\title{
Near-Wall Plasma Properties and EEDF Measurements of a 6-kW Hall Thruster
}

\author{
Rohit Shastry ${ }^{1}$, and Alec D. Gallimore ${ }^{2}$ \\ Plasmadynamics and Electric Propulsion Laboratory, University of Michigan, Ann Arbor, MI 48109 \\ and \\ Richard R. Hofer ${ }^{3}$ \\ Jet Propulsion Laboratory, California Institute of Technology, Pasadena, CA 91109
}

\begin{abstract}
In preparation for in-depth internal wall measurements for erosion and electron mobility studies, a flush-mounted Langmuir probe and emissive probe were used to characterize the near-wall region near the exit plane of a 6-kW Hall thruster. Various plasma properties and electron energy distribution functions were measured at discharge voltages of 150 and 300 $\mathrm{V}$, and anode mass flow rates of 10,20 , and $30 \mathrm{mg} / \mathrm{s}$, as well as at $600 \mathrm{~V}$ and $10 \mathrm{mg} / \mathrm{s}$. It was found that this region is characterized by a significantly lower number density and plasma potential than expected, while the electron temperature is relatively consistent with previous internal measurements. The electron energy distribution function was found to be highly non-Maxwellian, possibly due to filtering out of low-energy particles by large radial potential gradients. Incident ion power deposition and implications of the structure of the distribution function are discussed.
\end{abstract}

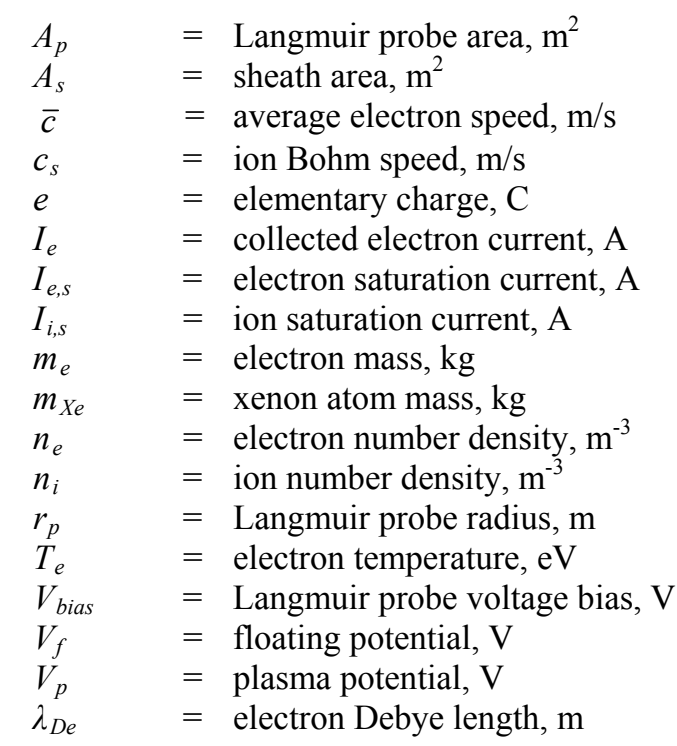

\section{Nomenclature}

\footnotetext{
${ }^{1}$ Ph.D. Candidate, Plasmadynamics and Electric Propulsion Laboratory, rshastry@umich.edu, AIAA student member.

${ }^{2}$ Arthur F. Thurnau Professor of Aerospace Engineering and Director of the Plasmadynamics and Electric Propulsion Laboratory, alec.gallimore@umich.edu, AIAA Associate Fellow.

${ }^{3}$ Technical Staff Member, Electric Propulsion Group, 4800 Oak Grove Dr., MS 125-109, Pasadena, CA 91109, richard.r.hofer@jpl.nasa.gov, AIAA senior member.
}

American Institute of Aeronautics and Astronautics 


\section{Introduction}

$\mathrm{H}$ all thruster lifetime models currently in development aim to provide predictive tools that would eliminate or substantially reduce the need to perform long duration, expensive life tests of flight articles [1-3]. A critical part of these modeling efforts is the need to understand and characterize the interaction between the plasma and the discharge channel walls. This interaction is composed of several complex processes that are not fully understood, and yet significantly affect thruster performance and lifetime. Several notable advances have been made in recent years in understanding plasma-wall interactions in Hall thrusters [4-6], but there is presently a noticeable lack of experimental validation. In particular, measurements of ion current and power incident on the ceramic walls as well as characterization of the electron energy spectrum would greatly contribute to the understanding of Hall thruster sheath physics, as well as provide validation and/or allow refinement of existing sheath models [7-10].

As a preparatory study, plasma properties and electron energy distribution functions (EEDFs) were measured near the wall of a $6-\mathrm{kW}$ Hall thruster. A boron nitride tab instrumented with a flush-mounted Langmuir probe was clamped in place downstream of the thruster exit plane, and data were taken over seven operating conditions at various discharge voltages and anode mass flow rates. Similarly, a swept emissive probe was used to provide an accurate, independent measurement of plasma potential under the same operating conditions. These data were then compared to internal measurements taken by Reid et al. using a high-speed axial reciprocating probe [11], in order to characterize any differences associated with the near-wall region.

The paper is organized as follows: Section II describes the experimental setup and data analysis techniques used in the study. Section III presents results of plasma potential, number density, electron temperature, and EEDFs for all operating conditions. Implications of the trends seen in ion power deposition and features of the local EEDF are discussed in Section IV, while Section V summarizes the pertinent results and conclusions of the study.

\section{Experimental Apparatus}

\section{A. Vacuum Facility}

Experiments were performed in the Large Vacuum Test Facility (LVTF) at the University of Michigan's Plasmadynamics and Electric Propulsion Laboratory (PEPL). The LVTF is a 6-m-diameter by 9-m-long cylindrical stainless steel chamber, equipped with seven CVI model TM-1200 cryopumps and liquid-nitrogen-cooled shrouds capable of providing a pumping speed of $240,000 \mathrm{l} / \mathrm{s}$ on xenon. Facility pressure was monitored with two commercially available hot-cathode ionization gauges, and a base pressure of $4 \times 10^{-7}$ torr was routinely achieved. At a total mass flow rate of $21.4 \mathrm{mg} / \mathrm{s}$, the facility pressure was $1.5 \times 10^{-5}$ torr, corrected for xenon.

\section{B. Hall Thruster}

The test article for this study was a 6-kW laboratory Hall thruster with an approximate throttling range of 100$600 \mathrm{mN}$ thrust and 1000-3000 s specific impulse. Research grade xenon propellant (99.999\% pure) was supplied using commercially available controllers with $\pm 1 \%$ full-scale accuracy. The controllers were calibrated before the experiment using a constant volume method that corrected for compressibility effects. The thruster is instrumented with a center-mounted $\mathrm{LaB}_{6}$ hollow cathode, whose flow rate was maintained at $7 \%$ of the anode flow rate. The discharge was sustained with a $100-\mathrm{kW}$ power supply capable of outputting $1000 \mathrm{~V}$ and $100 \mathrm{~A}$. Separate supplies were used to power the magnets, along with the cathode heater and keeper.

\section{Langmuir Probe}

A single Langmuir probe flush-mounted to a boron nitride wall was used to interrogate the near-wall region of the 6-kW Hall thruster. This probe was selected due to its simplicity and ability to measure several plasma properties such as number density, electron temperature, floating and plasma potentials, and EEDFs. However, the analysis of Langmuir probe data in order to obtain these properties can be complex due to various effects causing non-ideal behavior, leading to many different analysis techniques. Therefore, the following section will detail the data analysis used in this study. This will be followed by a description of the probe design and data acquisition.

\section{Data Analysis Techniques}

Most of the analysis methods used in this study follow simple Langmuir probe theory [12]. First, the floating potential was found by locating the voltage at which the collected current was zero. Next, the ion current was added to the collected current and a semilog plot of the resulting electron current was created from the I-V characteristic. A line was fit to the transition region of this plot, whose inverse slope corresponds to the electron temperature in $\mathrm{eV}$. 
Plasma potential was found using two different methods. First, a line was fit to the electron saturation region of the semilog plot. The intersection of this line and the line fit to the transition region corresponded to the plasma potential and the electron saturation current. Second, two derivatives were taken of a smoothed trace of the I-V characteristic. The voltage at which the second derivative is zero was taken as another measurement of plasma potential, and the current collected at this voltage was taken as the electron saturation current. Regardless of which technique was used, the electron saturation current was assumed to be from the thermal flux of electrons incident on the probe surface, given by

$$
I_{e, s}=\frac{1}{4} e n_{e} \bar{c} A_{p}=\frac{1}{4} e n_{e} \sqrt{\frac{8 e T_{e}}{\pi m_{e}}} A_{p} .
$$

With the electron saturation current and the electron temperature, the electron density can be determined.

Ion number density was similarly determined from the ion saturation current. However, due to the size of the probe used, sheath expansion effects made it difficult to determine the proper saturation current. Due to the planar geometry of the probe, Sheridan's model was used to quantify the sheath expansion effects and recover the proper ion saturation current [13]. This model provides empirical formulas (based on PIC simulations) for the area expansion based on the non-dimensional relative probe bias and probe radius:

$$
\frac{A_{s}}{A_{p}}=1+a \eta_{p}{ }^{b}, \eta_{p}=\frac{\left(V_{p}-V_{\text {bias }}\right)}{T_{e}}, a=2.28 \rho_{p}^{-0.749}, b=0.806 \rho_{p}^{-0.0692}, \rho_{p}=\frac{r_{p}}{\lambda_{D e}} .
$$

Thus, using the calculated plasma potential and electron temperature, the area expansion can be accounted for. Note that the model contains the electron Debye length, which in turn requires a plasma density. Use of this model is therefore an iterative process until a self-consistent solution is found. It should be noted that Sheridan's model was derived for a non-flowing, thermal plasma. Thus, its application here is intended as a first order correction that does not account for the directed ion energy present in Hall thrusters. Figure 1 shows the effects of the model on the ion saturation branch. After the sheath area correction, the saturation branch becomes much flatter, allowing for a more straightforward determination of the proper current. The saturation current was taken as the average current between the lowest probe bias and one electron temperature below the floating potential. If this current is assumed to be the Bohm current, it can be used to determine ion number density:

$$
I_{i, s}=0.61 e n_{i} c_{s} A_{p}=0.61 e n_{i} \sqrt{\frac{e T_{e}}{m_{X e}}} A_{p} .
$$

It was found that after sheath expansion corrections, the calculated ion and electron densities were in excellent agreement.

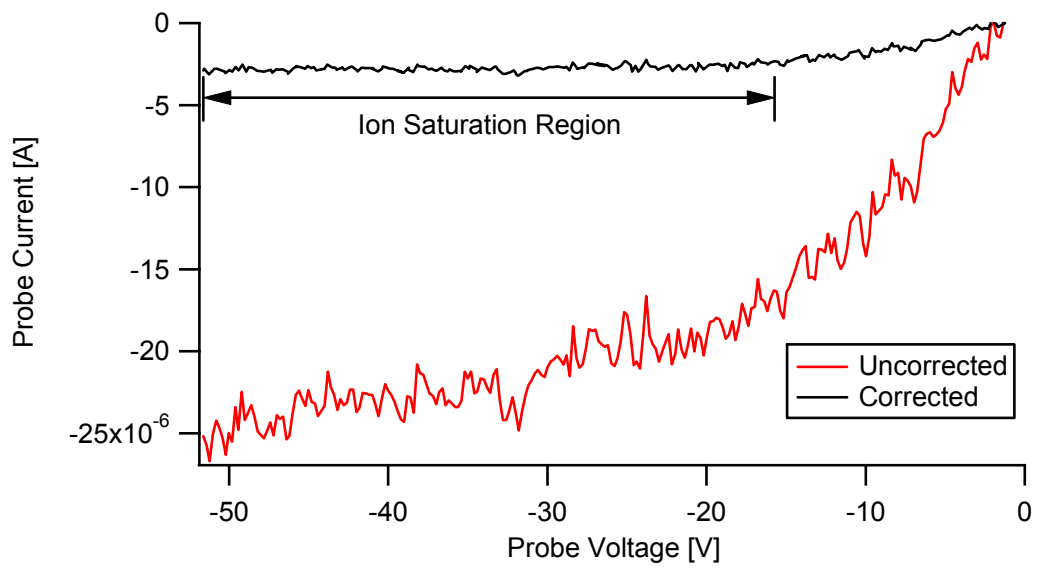

Figure 1: Comparison of the ion saturation branch of a Langmuir probe trace before and after correcting for sheath expansion effects using Sheridan's model. Note that after correction, the ion saturation region is much flatter. 
Electron energy distribution functions were calculated using the Druyvesteyn formula [14]:

$$
f(E)=\frac{2 m_{e}}{e^{2} A_{p}}\left(\frac{2 e\left(V_{p}-V_{\text {bias }}\right)}{m_{e}}\right)^{1 / 2}\left(\frac{d^{2} I_{e}}{d V_{\text {bias }}^{2}}\right) .
$$

The second derivative of a smoothed I-V characteristic was taken, and was used along with the plasma potential measurement to determine the local EEDF. It should be noted that integration of the above EEDF gives another calculation of plasma number density, and that an effective temperature can be calculated for the distribution, $(2 / 3)$ $<\varepsilon>$, where $<\varepsilon>$ is the average electron energy based on the EEDF.

\section{Probe Design}

Since this particular study is a preparatory experiment for internal flush-mounted diagnostics, the probe design attempts to emulate such an environment in order to collect a representative data set. A 0.381-mm-diameter tungsten probe was fit through a 1.6-mm-diameter alumina tube. This assembly was then placed within a boron nitride tab such that the probe tip was flush with the tab surface (see Figure 2).
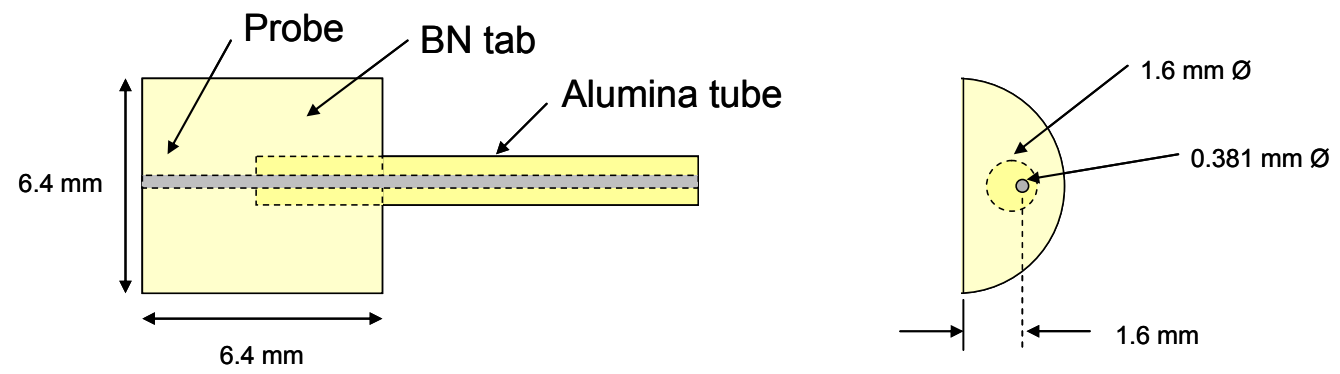

Figure 2: Schematic drawing of flush-mounted Langmuir probe used in study. Note: note to scale.

The probe was then mounted onto the thruster face using a stainless steel mounting block and clamp, such that the flat portion of the tab lay adjacent to the outer wall exit plane. The final position of the probe was roughly $1.6 \mathrm{~mm}$ downstream of the exit plane and $1 \mathrm{~mm}$ radially away from the outer wall radius (plasma side, see Figure 3 ). It should be noted that this location is not completely representative of conditions at the channel walls, since directed ion energies will typically be higher while number densities and electron temperatures will be lower than those internal to the channel. However, this position was chosen because the magnetic field properties are representative of what can be found along the inner and outer channel walls, which was deemed more important due to its impact on the collected data.

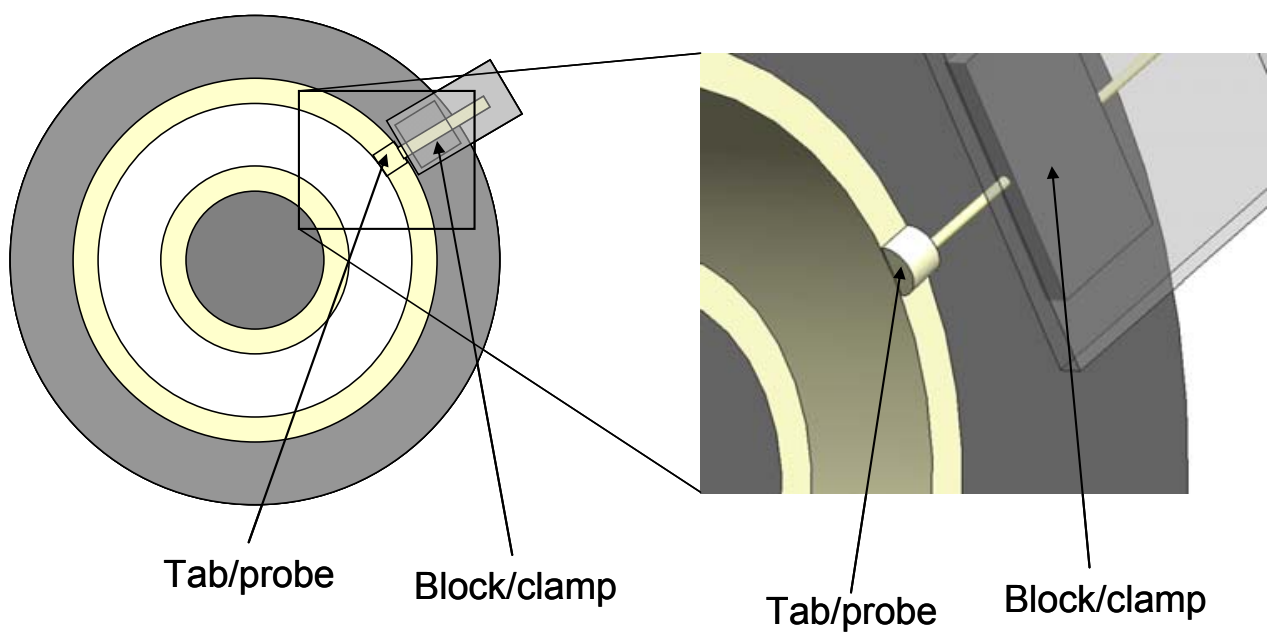

Figure 3: Schematic of Langmuir probe placement with respect to the thruster body. Note: not to scale. 


\section{Data Acquisition}

Probe current and voltage were collected and stored using a data acquisition system (DAQ) capable of a $500 \mathrm{kHz}$ scan rate across all channels. Figure 4 shows a schematic of the measurement circuit used for the Langmuir probe. A function generator was connected to a bipolar power supply, which supplied the desired voltage waveform to the probe. The current shunt was comprised of a single $100 \Omega$ thin film resistor, while the voltage divider used to measure the applied voltage was comprised of $8.18 \mathrm{M} \Omega$ and $0.24 \mathrm{M} \Omega$ thin film resistors. The signals were isolated from the DAQ with voltage-following isolation amplifiers, each having a bandwidth of $20 \mathrm{kHz}$. Blocking diodes were placed in parallel with the current shunt in order to protect the isolation amplifier from large current spikes. The circuit was calibrated at DC prior to the study, and displayed excellent linearity. The probe was biased using a symmetric triangle wave with a

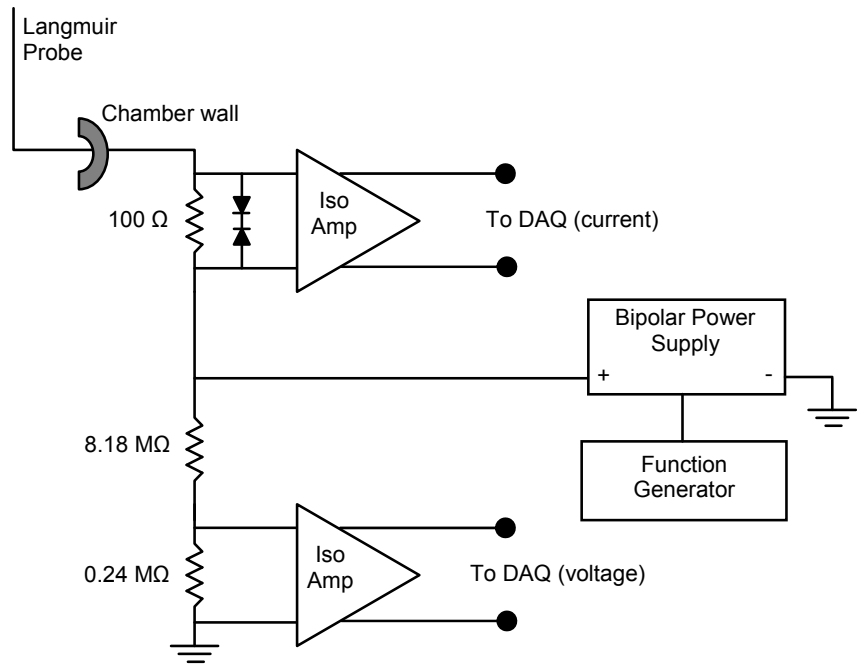

Figure 4: Electrical diagram of Langmuir probe data collection circuit. frequency of 20-30 Hz, well within the bandwidth of the isolation amplifiers. Each channel was scanned at 40-50 $\mathrm{kHz}$, resulting in approximately 1000 points per I-V characteristic. Capacitive effects were characterized by taking data with no plasma present, and were subtracted out in post processing. Two hundred traces were taken at each operating condition and averaged before any data analysis was performed.

\section{Emissive Probe}

While the single Langmuir probe offers the ability to measure several important plasma properties, its ability to determine plasma potential accurately can be reduced substantially by non-ideal conditions. The presence of beams, magnetic fields, and significant plasma oscillations can all cause error in the determination of plasma potential [12]. For this reason, an emissive probe was used as an additional measurement of plasma potential. The floating emissive probe has extensive heritage as a diagnostic for internal plasma potential measurements in Hall thrusters $[11,15,16]$. Floating emissive probes rely on a hot filament to thermionically emit electrons, which raises the probe's floating potential. As emission increases, the floating potential approaches the local plasma potential. However, space charge limitations on probe emission prevent the floating potential from properly reaching the plasma potential. This requires the use of a sheath model, which is dependent on the local electron temperature, to correct for space charge limitations. Thus, the floating emissive probe typically requires a Langmuir probe as a complementary diagnostic to measure plasma potential. Furthermore, uncertainties with the sheath model and its use in Hall thrusters create uncertainty in the measurement on the order of the local electron temperature [16].

Since an independent measurement of plasma potential was desired, the swept emissive probe was utilized in this study. While this diagnostic is more time-consuming and difficult to implement than the floating emissive probe, the stationary nature of a near-wall probe allowed for its use with minimal added complexity. The swept emissive probe is run similarly to a Langmuir probe, except it has the ability to emit thermionic electrons. I-V characteristics are taken at various levels of electron emission; this emission shows up as increased ion current in the characteristic. However, once the probe is biased above the plasma potential, all emission should be reflected back to the probe surface, resulting in zero effective emission. Thus, the voltage at which all I-V characteristics begin to overlap is an accurate measurement of the local plasma potential [17]. While this diagnostic is significantly more complex than the floating emissive probe, it allows for an independent, highly accurate measurement of plasma potential.

\section{Probe Design}

The emissive probe used in this study is based on the design primarily from Haas [15], but augmented by Linnell and Reid [11,16]. A 0.127 -mm-diameter filament made of $1 \%$ thoriated tungsten was placed within a 1.6 -mmdiameter double-bored alumina tube. Thoriated tungsten was chosen as the filament material due to its higher emission capabilities; this translates to a lower operating temperature for the required emission currents, thus bringing the filament further away from its melting point. The filament was bent into a loop with a 0.5 -mm radius of curvature, and brought into contact with 0.254-mm-diameter copper lead wires. Tungsten filler wires were then 
placed inside the tube to provide a tight fit between the filament and leads, as well as excellent electrical contact (see Figure 5). The probe was mounted to the thruster using a stainless steel block and clamp, similar to the Langmuir probe used in this study. The final position of the probe tip was approximately $1.4 \mathrm{~mm}$ from the outer wall radius, and $2.6 \mathrm{~mm}$ beyond the exit plane, which was within $1 \mathrm{~mm}$ of the location of the Langmuir probe.

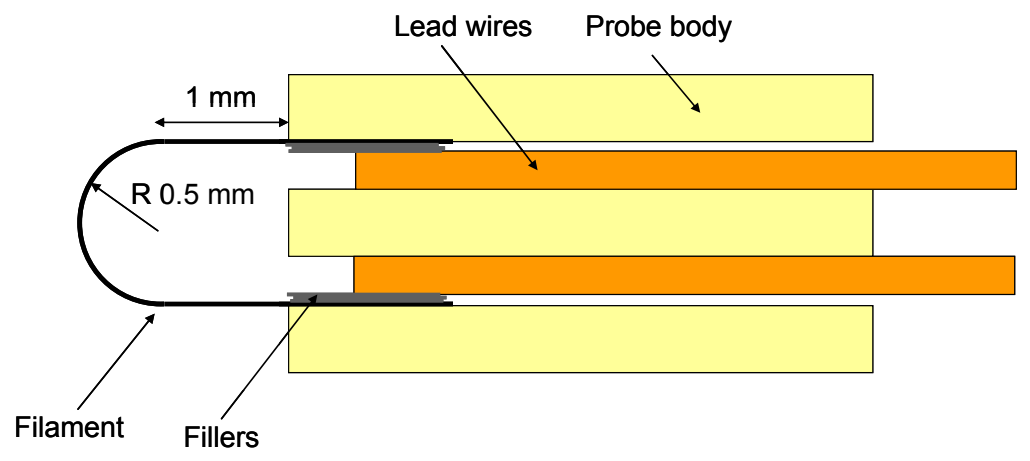

Figure 5: Schematic drawing of emissive probe used in study. Note: note to scale.

\section{Data Acquisition}

Due to the nature of the swept emissive probe, data was collected in a similar manner to the single Langmuir probe. Probe current and voltage were collected and stored using a data acquisition system (DAQ) which scanned each channel at $40 \mathrm{kHz}$. Figure 6 shows a schematic of the measurement circuit used for the swept emissive probe. The circuit was calibrated at DC prior to the study, and displayed excellent linearity. A floating power supply was used to provide the filament current required for thermionic emission. Current was slowly increased from zero while the filament was optically monitored. Once the probe showed noticeable signs of emission, I-V characteristics were taken at 2-3 different levels of emission current. A characteristic was taken at zero filament current as well. The probe was biased using a symmetric triangle wave with a frequency of $20 \mathrm{~Hz}$, resulting in approximately 1000 points per I-V characteristic. The probe bias was applied in between a voltage divider

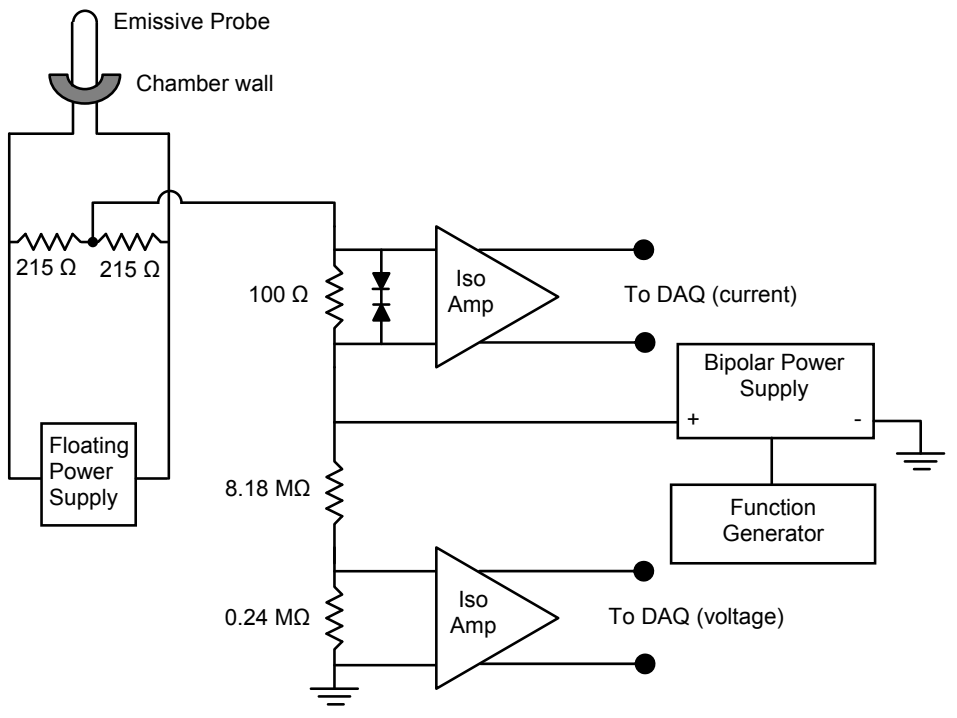

Figure 6: Electrical diagram of the emissive probe data collection circuit.

placed in parallel with the filament. This voltage divider was comprised of two $215 \Omega$ thin film resistors, which was used to apply probe bias at the center of the filament where most emission should occur [17]. This was done to minimize uncertainties associated with the voltage drop across the filament. Capacitive effects were characterized by taking data with no plasma present, and were subtracted out in post processing. Furthermore, the voltage drop across the shunt was taken into account in data analysis when determining probe bias. While this correction is usually negligible, enough current was drawn by the probe to significantly affect a measurement which would otherwise have uncertainties of $\sim 1-2 \mathrm{~V}$. Two hundred traces were taken at each operating condition and averaged before any data analysis was performed.

\section{Results}

Langmuir probe and emissive probe data was taken near the outer wall exit plane of a 6-kW Hall thruster over a wide range of discharge voltages and anode mass flow rates. Discharge voltages of 150 and $300 \mathrm{~V}$, each at anode 
mass flow rates of 10, 20, and $30 \mathrm{mg} / \mathrm{s}$, are presented, as well as $600 \mathrm{~V}$ and $10 \mathrm{mg} / \mathrm{s}$. Emissive probe data at $300 \mathrm{~V}$ and $30 \mathrm{mg} / \mathrm{s}$ is not available due to probe failure at this operating condition. Since this study is in preparation for erosion and electron mobility investigations to be done inside the channel, properties relevant to these measurements will be presented here. In particular, plasma potential, as well as the sheath potential drop, ion current and density, and EEDF measurements are discussed.

\section{A. Plasma Potential}

The local plasma potential is an important measurement in this study, as it is necessary to determine the sheath potential drop, properly correct the ion saturation branch in Langmuir probe data using Sheridan's model, and determine the reference potential for the calculation of EEDFs. Due to its importance, the plasma potential was calculated using four different methods. The line extrapolation and derivative methods for Langmuir probes, as well as the swept emissive probe, have already been discussed in Section II. The fourth method is to use the floating potential and shift it by an appropriate factor using the electron temperature [12]:

$$
V_{p}=V_{f}+\zeta T_{e}
$$

In simple Langmuir probe theory, $\zeta=5.77$ for xenon. While this is not the most robust method to determine plasma potential, it offers a rough estimate that can be compared to other measurements.

Figure 7 shows a typical result of the line extrapolation and derivative methods using Langmuir probe data taken at $300 \mathrm{~V}, 20 \mathrm{mg} / \mathrm{s}$. These two methods usually agree with each other to within a volt for data taken in the far-field plume [18]. However, it is evident from the figure that these two methods are not in good agreement, differing by approximately $30 \mathrm{~V}$. Given the non-ideal environment of the interrogated region, it is not surprising to have such uncertainty in the plasma potential measurement of Langmuir probe data. This is further evidence of the need for a more robust, accurate determination of plasma potential, which is why the swept emissive probe was used. It should be noted that a distinct peak in the first derivative (or, equivalently, a zero in the second derivative) was usually not found, and in these cases the plasma potential was calculated by extrapolating the shape of the second derivative to its zero crossing.

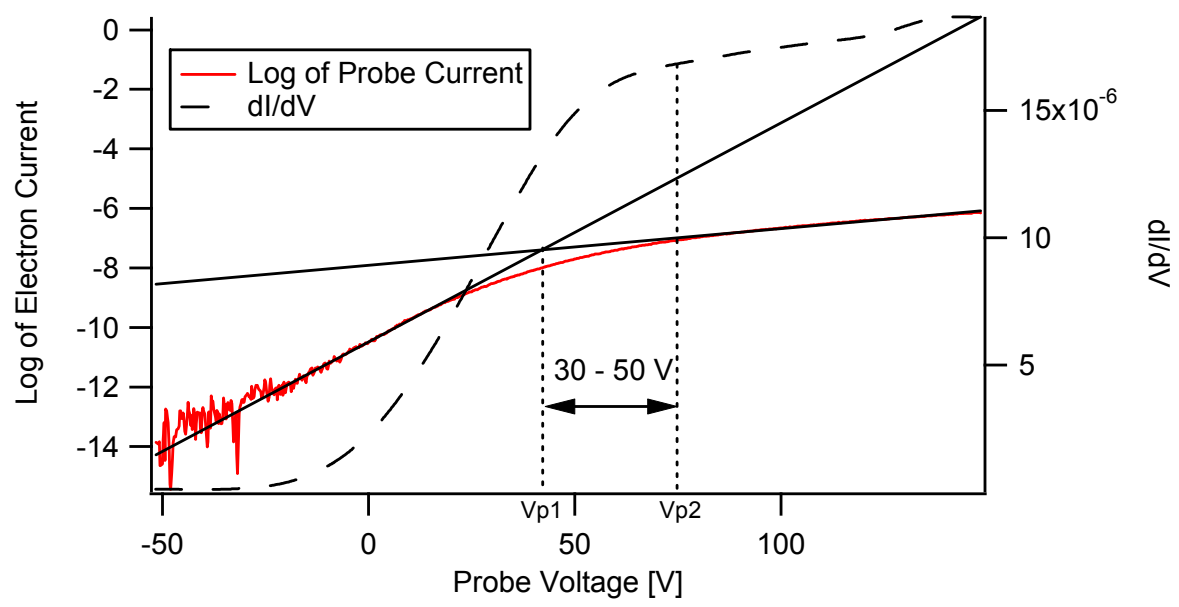

Figure 7: Graphical comparison of the resulting plasma potentials from Langmuir probe data using two different analysis methods. Differences between these methods ranged from $30-50 \mathrm{~V}$, depending on operating condition.

Figure 8 shows a typical plot of swept emissive probe data, also taken at $300 \mathrm{~V}, 20 \mathrm{mg} / \mathrm{s}$. As the filament current is increased, there is a noticeable increase in the collected ion current. Once the I-V characteristics begin to overlap (within the noise level), the probe bias is taken to be above the plasma potential. It should be noted that sometimes the cold trace did not perfectly overlap with the other traces. This could possibly be due to impurities causing changes in the material work function [17]. Because of this, only the warm and hot traces were typically used to determine plasma potential. Due to the inherent noise level of the data, an uncertainty of approximately $3 \mathrm{~V}$ in the plasma potential was estimated for most operating conditions, while at $600 \mathrm{~V}$ and $10 \mathrm{mg} / \mathrm{s}$ the uncertainty was found to be approximately $15 \mathrm{~V}$. 


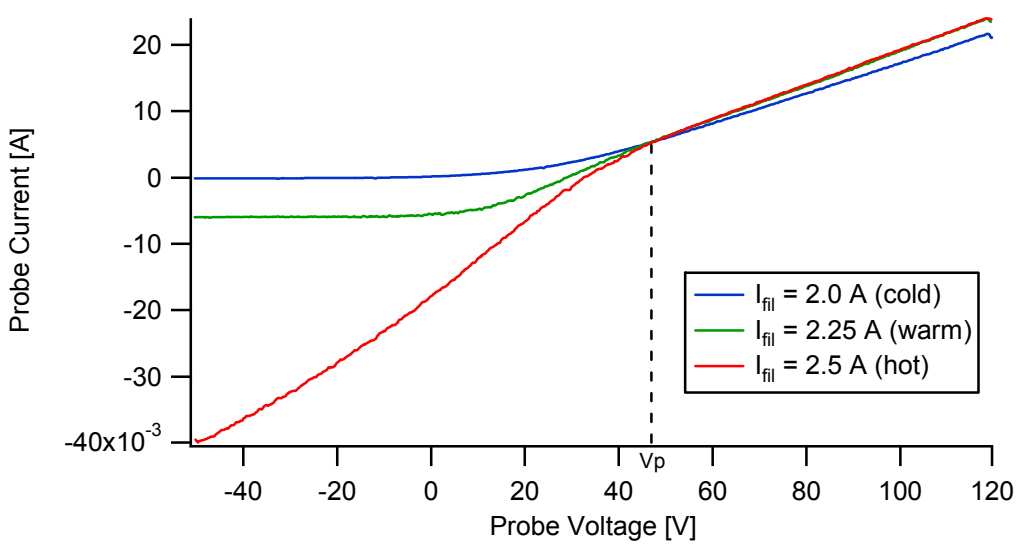

Figure 8: Typical I-V characteristics from swept emissive probe at various filament heater currents. The probe voltage at which the traces begin to overlap is taken as the plasma potential.

In order to compare this measurement with those from the Langmuir probe, the swept emissive probe data was corrected for the location difference between probes. While this location difference was kept within approximately $1 \mathrm{~mm}$, this could create a potential difference of tens of volts if within the acceleration zone of the thruster. The location correction was done using the floating potential and electron temperature measured with the emissive probe at zero emission. Using Equation (5) for both the Langmuir probe and emissive probe, while leaving $\zeta$ and $\mathrm{V}_{\mathrm{p}}$ for the Langmuir probe as variables, an estimate of the plasma potential at the location of the Langmuir probe could be calculated:

$$
V_{p_{1}}=V_{f_{1}}+\left(V_{p_{2}}-V_{f_{2}}\right) \frac{T_{e_{1}}}{T_{e_{2}}} .
$$

In this equation, a subscript of " 1 " corresponds to the location of the Langmuir probe, while a subscript of "2" corresponds to the location of the emissive probe. Equation (6) assumes that the two locations were close enough such that $\zeta$ was constant.

Table 1 compares the four different methods of determining plasma potential across all the operating conditions tested. Since the emissive probe is considered to be the most robust measurement of plasma potential, all other methods were compared to its data. On average, the linear extrapolation method had a relative difference of $49 \%$, while the derivative method had a relative difference of only $12 \%$. The shifted floating potential method had a relative difference of $13 \%$ when compared to the emissive probe data, making it comparable to the derivative method. When considering individual data sets, the derivative method is the most accurate method to determine plasma potential from Langmuir probe data, and will be used in subsequent Langmuir probe data analysis.

\begin{tabular}{|c|c|c|c|c|}
\hline $\begin{array}{c}\text { Operating } \\
\text { Condition }\end{array}$ & $\begin{array}{c}\text { Corrected Emissive } \\
\text { Probe Data (V) }\end{array}$ & $\begin{array}{c}\text { Line Extrapolation } \\
\text { Method (V) }\end{array}$ & $\begin{array}{c}\text { Derivative Method } \\
\text { (V) }\end{array}$ & $\begin{array}{c}\text { Shifted Floating } \\
\text { Potential Method (V) }\end{array}$ \\
\hline $150 \mathrm{~V}, 10 \mathrm{mg} / \mathrm{s}$ & 63 & $34(46)$ & $65(4)$ & $55(13)$ \\
\hline $150 \mathrm{~V}, 20 \mathrm{mg} / \mathrm{s}$ & 70 & $35(50)$ & $65(8)$ & $57(19)$ \\
\hline $150 \mathrm{~V}, 30 \mathrm{mg} / \mathrm{s}$ & 63 & $34(47)$ & $61(3)$ & $48(24)$ \\
\hline $300 \mathrm{~V}, 10 \mathrm{mg} / \mathrm{s}$ & 70 & $36(49)$ & $88(26)$ & $82(16)$ \\
\hline $300 \mathrm{~V}, 20 \mathrm{mg} / \mathrm{s}$ & 84 & $42(50)$ & $75(11)$ & $76(9)$ \\
\hline $300 \mathrm{~V}, 30 \mathrm{mg} / \mathrm{s}$ & $\mathrm{N} / \mathrm{A}$ & 44 & 83 & 82 \\
\hline $600 \mathrm{~V}, 10 \mathrm{mg} / \mathrm{s}$ & 154 & $71(54)$ & $119(23)$ & $154(0)$ \\
\hline
\end{tabular}

Table 1: Comparison of measured plasma potential using four different measurement and analysis methods. Percent differences when compared to the corrected emissive probe data are given in parentheses. On average, the derivative method gives the most accurate plasma potential using Langmuir probe data. 
Once the plasma potential was determined, the effective sheath potential drop between the plasma and the wall was found for each operating condition. The measured potential drop, $V_{p}-V_{f}$, was corrected for secondary electron emission (SEE) effects using the model described in Refs. [2] and [19]. SEE effects were assumed to be negligible for the tungsten probe due to its low emission coefficients [18]. The correction allows the sheath potential drop at the adjacent boron nitride wall to be calculated. Table 2 shows this drop for all operating conditions tested, in units of volts and electron temperatures (only electron temperatures measured by the flush-mounted Langmuir probe were used).

\begin{tabular}{|c|c|c|}
\hline $\begin{array}{c}\text { Operating } \\
\text { Condition }\end{array}$ & $\begin{array}{c}\text { Sheath Potential } \\
\text { Drop (V) }\end{array}$ & $\begin{array}{c}\text { Sheath } \\
\text { Potential Drop } \\
\mathbf{( T}_{\mathbf{e}} \text { ) }\end{array}$ \\
\hline $150 \mathrm{~V}, 10 \mathrm{mg} / \mathrm{s}$ & 50 & 5.6 \\
\hline $150 \mathrm{~V}, 20 \mathrm{mg} / \mathrm{s}$ & 50 & 5.2 \\
\hline $150 \mathrm{~V}, 30 \mathrm{mg} / \mathrm{s}$ & 53 & 5.8 \\
\hline $300 \mathrm{~V}, 10 \mathrm{mg} / \mathrm{s}$ & 60 & 4.3 \\
\hline $300 \mathrm{~V}, 20 \mathrm{mg} / \mathrm{s}$ & 51 & 3.7 \\
\hline $300 \mathrm{~V}, 30 \mathrm{mg} / \mathrm{s}$ & 55 & 3.6 \\
\hline $600 \mathrm{~V}, 10 \mathrm{mg} / \mathrm{s}$ & 8.2 & 0.28 \\
\hline
\end{tabular}

Table 2: Calculated sheath potential drop, in units of volts and local electron temperatures, for all operating conditions tested.

It is interesting to note that at discharge voltages of 150 and $300 \mathrm{~V}$, the effective sheath potential drop is approximately constant at $50-60 \mathrm{~V}$. The sheath potential drop was found to be substantially lower at $600 \mathrm{~V}$ and 10 $\mathrm{mg} / \mathrm{s}$ despite the large increase in electron temperature. This is because the predicted secondary electron emission at the wall is space charge limited, resulting in a potential drop of approximately $1 \mathrm{~T}_{\mathrm{e}}$. The sheath potential actually decreases with discharge voltage when non-dimensionalized by electron temperature, partly due to increased SEE effects. These trends will have important implications for the ion energy gained in the sheath and incident power deposition, which will be discussed in Section IV.

\section{B. Ion Current Density and Number Density}

Langmuir probe data was taken at several operating conditions, and the ion saturation branch was used to determine incident ion current density and number density. Sheridan's model [13] was used to correct for sheath expansion and the resulting ion current was assumed to be equal to the Bohm current in order to determine ion density. It should be noted that this assumption is not valid if there is a significant ion drift velocity towards the probe. However, the magnetic field is this region is nearly radial; this indicates that the electric fields, and thus the ion drift velocities, are likely axial and thus parallel to the probe surface. Table 3 gives the results at all operating conditions tested.

\begin{tabular}{|c|c|c|}
\hline Operating Condition & Incident Ion Current Density (A/m $\left.\mathbf{~}^{2}\right)$ & Ion Number Density $\left.\mathbf{( m}^{-3}\right)$ \\
\hline $150 \mathrm{~V}, 10 \mathrm{mg} / \mathrm{s}$ & 49 & $1.2 \times 10^{17}$ \\
\hline $150 \mathrm{~V}, 20 \mathrm{mg} / \mathrm{s}$ & 188 & $5.3 \times 10^{17}$ \\
\hline $150 \mathrm{~V}, 30 \mathrm{mg} / \mathrm{s}$ & 157 & $4.5 \times 10^{17}$ \\
\hline $300 \mathrm{~V}, 10 \mathrm{mg} / \mathrm{s}$ & 13 & $2.3 \times 10^{16}$ \\
\hline $300 \mathrm{~V}, 20 \mathrm{mg} / \mathrm{s}$ & 38 & $7.9 \times 10^{16}$ \\
\hline $300 \mathrm{~V}, 30 \mathrm{mg} / \mathrm{s}$ & 80 & $1.5 \times 10^{17}$ \\
\hline $600 \mathrm{~V}, 10 \mathrm{mg} / \mathrm{s}$ & 9.1 & $1.2 \times 10^{16}$ \\
\hline
\end{tabular}

Table 3: Calculated ion current density and number density at tested operating conditions. These values tend to increase with flowrate and decrease with discharge voltage.

From Table 3, it is clear that for a given anode flow rate, the ion number density decreases with increasing discharge voltage. This could be due either to improved beam focusing at higher discharge voltages, thus decreasing the density near the walls; or from the acceleration zone receding further upstream with increasing discharge voltage, causing decreased ion density due to higher local ion velocities. Due to the fact that this study consists of a singlepoint measurement, it is difficult to determine which one of these factors is responsible for this trend. It is 
interesting to note that the incident ion current exhibits the same trend as the ion density, which will become important when ion power deposition to the wall is discussed in Section IV.

\section{Electron Energy Distribution Functions}

Using the Druyvesteyn formula, EEDFs were determined from the Langmuir probe data using the second derivative of the characteristic and the plasma potential. Figure 9 shows a typical EEDF at $300 \mathrm{~V}, 20 \mathrm{mg} / \mathrm{s}$, as well as the equivalent Maxwellian distribution given the measured electron temperature. From the figure, it is evident that the distribution is highly non-Maxwellian. The peak (most probable) energy is much higher than predicted by the electron temperature. Table 4 compares the electron temperature measured using simple Langmuir probe theory to the effective electron temperature found using the measured EEDF.

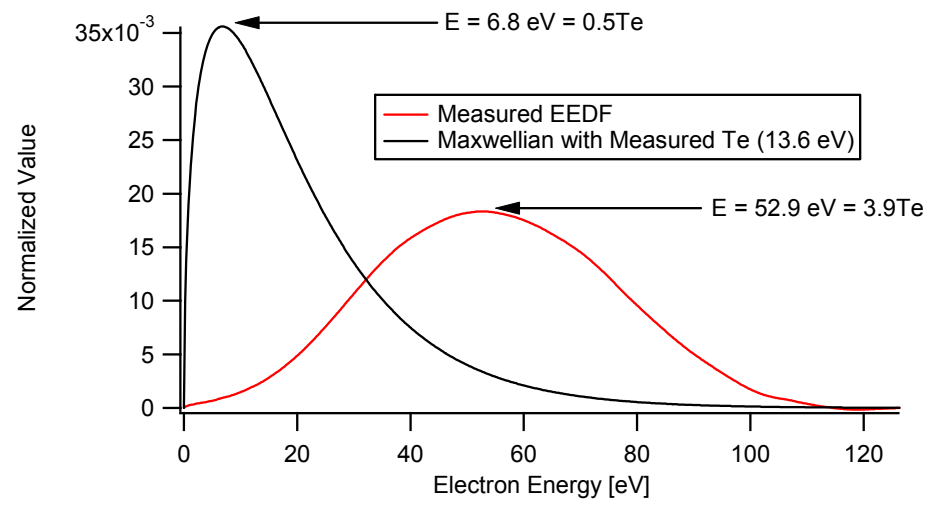

Figure 9: Representative EEDF measured near the wall, compared to the expected Maxwellian distribution given the measured electron temperature. The average electron energy appears to be significantly higher than a Maxwellian distribution would predict.

\begin{tabular}{|c|c|c|}
\hline Operating Condition & $\mathbf{T}_{\mathbf{e}}(\mathbf{e V})$ & $\mathbf{T}_{\mathbf{e}, \text { eff }}(\mathbf{e V})$ \\
\hline $150 \mathrm{~V}, 10 \mathrm{mg} / \mathrm{s}$ & 8.9 & 29 \\
\hline $150 \mathrm{~V}, 20 \mathrm{mg} / \mathrm{s}$ & 9.6 & 28 \\
\hline $150 \mathrm{~V}, 30 \mathrm{mg} / \mathrm{s}$ & 9.1 & 29 \\
\hline $300 \mathrm{~V}, 10 \mathrm{mg} / \mathrm{s}$ & 14 & 45 \\
\hline $300 \mathrm{~V}, 20 \mathrm{mg} / \mathrm{s}$ & 14 & 37 \\
\hline $300 \mathrm{~V}, 30 \mathrm{mg} / \mathrm{s}$ & 15 & 43 \\
\hline $600 \mathrm{~V}, 10 \mathrm{mg} / \mathrm{s}$ & 29 & 70 \\
\hline
\end{tabular}

Table 4: Comparison of the measured electron temperature and calculated effective temperature based on the measured EEDF. Note that the effective temperature is approximately $3 \mathrm{X}$ higher than the measured temperature.

From Table 4, the effective electron temperature is roughly three times higher than the measured electron temperature. The reason for this discrepancy can be seen in Figure 10. The electron retarding branch of the Langmuir probe characteristic contains a linear region, indicative of a Maxwellian distribution, as well as a nonlinear region. The linear region is the source of the measured electron temperature using simple Langmuir probe theory. However, this region does not continue all the way to the plasma potential (as it should if the distribution were purely Maxwellian); less electron current is collected than expected based on the preceding Maxwellian distribution, shown by the extrapolation of the linear region. This indicates that a portion of the electron distribution may be Maxwellian described by the measured electron temperature, but the remaining distribution is not. Possible causes and implications of this non-Maxwellian distribution are discussed in more detail in Section IV. 


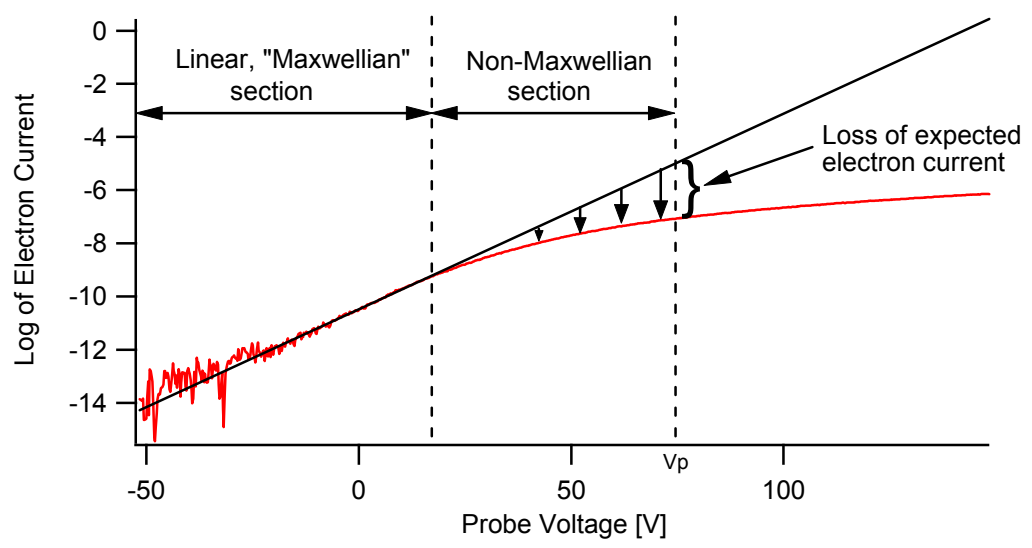

Figure 10: Semi-log plot of electron current distinguishing sections of Maxwellian and non-Maxwellian electron collection. Extrapolation of the linear, Maxwellian region shows that less current is collected near the plasma potential than expected.

The electron energy distributions were also compared across discharge voltages and mass flow rates. Figure 11 shows the EEDFs at $10 \mathrm{mg} / \mathrm{s}$ and 150,300 , and $600 \mathrm{~V}$, as well as at $300 \mathrm{~V}$ and 10,20 , and $30 \mathrm{mg} / \mathrm{s}$. As expected, the overall distribution tends to get wider and the peak shifts towards higher energies as discharge voltage increases, but does not change significantly with flow rate. These trends are reflected in both the measured and effective electron temperatures.
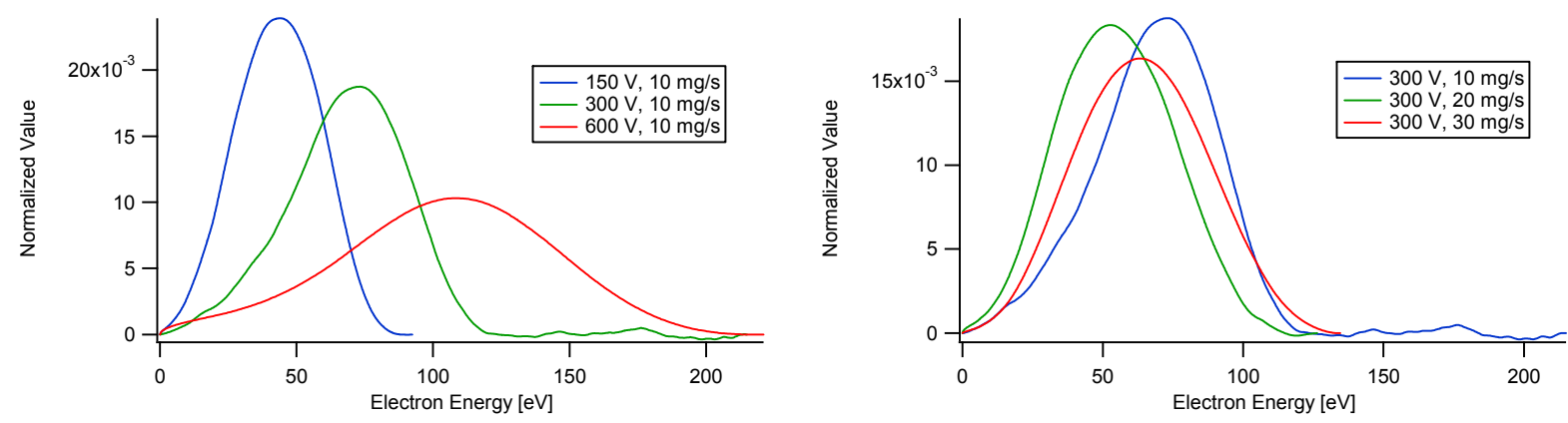

Figure 11: Comparison of EEDFs across operating conditions. Left: EEDF comparison at constant anode flow rate. Right: EEDF comparison at constant discharge voltage.

\section{Discussion}

Since this study was performed in preparation for internal measurements to aid understanding of erosion and electron mobility mechanisms, quantities relevant to these phenomena are discussed here. In particular, incident ion power deposition to the wall is compared across operating conditions. Plasma properties are compared to internal data taken by Reid et al. [11] in order to determine how the plasma discharge differs close to the wall. Finally, the structure of the local EEDF is discussed and implications are suggested.

\section{A. Ion Power Deposition}

Since erosion of the channel wall is directly related to the incident particle flux and energy, the ion power deposition is calculated and compared across all operating conditions tested. It has been shown that a significant amount of the power deposition from ions can come from the ion beam energy [2]. However, since this energy is not readily determined and the present study is focused on electron and sheath physics, only the contribution from the sheath is presented here. The incident power per unit area from the sheath is simply the product of the ion current density and the sheath potential drop. Table 5 gives these properties at each operating condition. 


\begin{tabular}{|c|c|c|c|}
\hline $\begin{array}{c}\text { Operating } \\
\text { Condition }\end{array}$ & $\begin{array}{c}\text { Ion Current Density } \\
\left(\mathbf{A} / \mathbf{m}^{\mathbf{2}}\right)\end{array}$ & $\begin{array}{c}\text { Sheath Potential Drop } \\
(\mathbf{V})\end{array}$ & $\begin{array}{c}\text { Power Deposition Density } \\
\left(\mathbf{W} / \mathbf{m}^{2}\right)\end{array}$ \\
\hline $150 \mathrm{~V}, 10 \mathrm{mg} / \mathrm{s}$ & 49 & 50 & $2.4 \times 10^{3}$ \\
\hline $150 \mathrm{~V}, 20 \mathrm{mg} / \mathrm{s}$ & 188 & 50 & $9.3 \times 10^{3}$ \\
\hline $150 \mathrm{~V}, 30 \mathrm{mg} / \mathrm{s}$ & 157 & 53 & $8.3 \times 10^{3}$ \\
\hline $300 \mathrm{~V}, 10 \mathrm{mg} / \mathrm{s}$ & 13 & 60 & $7.7 \times 10^{2}$ \\
\hline $300 \mathrm{~V}, 20 \mathrm{mg} / \mathrm{s}$ & 38 & 51 & $4.9 \times 10^{3}$ \\
\hline $300 \mathrm{~V}, 30 \mathrm{mg} / \mathrm{s}$ & 80 & 55 & $7.4 \times 10^{3}$ \\
\hline $600 \mathrm{~V}, 10 \mathrm{mg} / \mathrm{s}$ & 9.1 & 8.2 & $10^{1}$ \\
\hline
\end{tabular}

Table 5: Comparison of incident ion current density, sheath potential drop, and calculated ion power deposition at all operating conditions tested. Note that changes in power deposition are dominated by changes in ion current density.

While limited insight can be gained since measurements were taken at a single point, it is still evident from Table 5 that power deposition to the wall is maximized at low discharge voltages and high flow rates. This appears to be due mainly to the increased current density at the probe, possibly caused by larger beam divergences at lower discharge voltages. The trend in current density seems to dominate over any trends in sheath potential drop, making anode flow rate, ionization efficiency, and beam divergence key factors in ion power deposition. It is interesting to note that the power deposition is significantly lower at $600 \mathrm{~V}$ and $10 \mathrm{mg} / \mathrm{s}$ due to a lower ion current density and sheath potential drop. While this implies that the incident ion power is minimized at this operating condition, the ion beam energy, which will be significant at $600 \mathrm{~V}$, must also be taken into account when considering overall power deposition. Currently there is no clear trend between discharge power and the measured power deposition. However, conclusions cannot be drawn until a more complete spatial map of the incident ion density, energy, and power deposition is performed.

\section{B. Characterization of the Local Plasma}

Determining plasma properties close to the channel wall and comparing them to properties closer to channel centerline is important in gaining insight into erosion and electron mobility phenomena. Plasma potential, electron temperature and ion number density are compared between this study and data taken by Reid et al [11]. Internal measurements from Reid et

\begin{tabular}{|l|c|c|}
\hline & At flush probe & $\begin{array}{c}\mathbf{1 0 \%} \mathbf{L}_{\mathbf{w}} \text { from } \\
\text { outer wall }\end{array}$ \\
\hline Plasma Potential $(\mathrm{V})$ & 75 & 109 \\
\hline Electron Temperature $(\mathrm{eV})$ & 14 & 10 \\
\hline Ion Number Density $\left(\mathrm{m}^{-3}\right)$ & $7.8 \times 10^{16}$ & $1.1 \times 10^{18}$ \\
\hline
\end{tabular}

Table 6: Comparison of data taken near the wall to internal measurements taken by Reid et al. al. are taken along the same magnetic field line as the Langmuir probe used in this study, and located at $10 \%$ the channel width $\left(\mathrm{L}_{\mathrm{w}}\right)$ from the outer wall, the closest radial location interrogated in the study. Table 6 shows the comparison at the nominal condition of $300 \mathrm{~V}$ and $20 \mathrm{mg} / \mathrm{s}$. While there is relative agreement between the measured electron temperatures, Table 6 indicates a $34 \mathrm{~V}$ voltage drop between the two data sets, as well as a 93\% reduction in number density. Due to the similarity in electron temperature, the Boltzmann equation was used to determine whether the plasma potential and number density trends were consistent:

$$
n / n_{o}=\exp \left(\phi / T_{e}\right) .
$$

Using an averaged electron temperature and the measured ion density ratio, the expected potential drop is $32 \mathrm{~V}$, which is in excellent agreement with the measured potential drop. This indicates that large density drops near the channel walls correlate to large radial potential drops which are not evident in data taken closer to the channel centerline.

Given the relative agreement in electron temperatures between the two data sets, it is plausible that the same electron distribution is being sampled; the lower plasma density would indicate that less of the population is present closer to the wall. In order to test this possibility, the local EEDF is compared to a Maxwellian distribution given the local measured temperature. While this was shown previously, the local EEDF will now be scaled by the local number density while the Maxwellian distribution will be scaled by the density measured by Reid et al., given in Table 6. Figure 12 shows the comparison of these two distributions. The figure shows relative agreement between 
the two distributions at higher energies, which is the portion of the distribution which could overcome the large potential drop between the two regions. This indicates that the potential drop may be filtering out lower-energy electrons closer to the wall, increasing the average electron energy in this region.

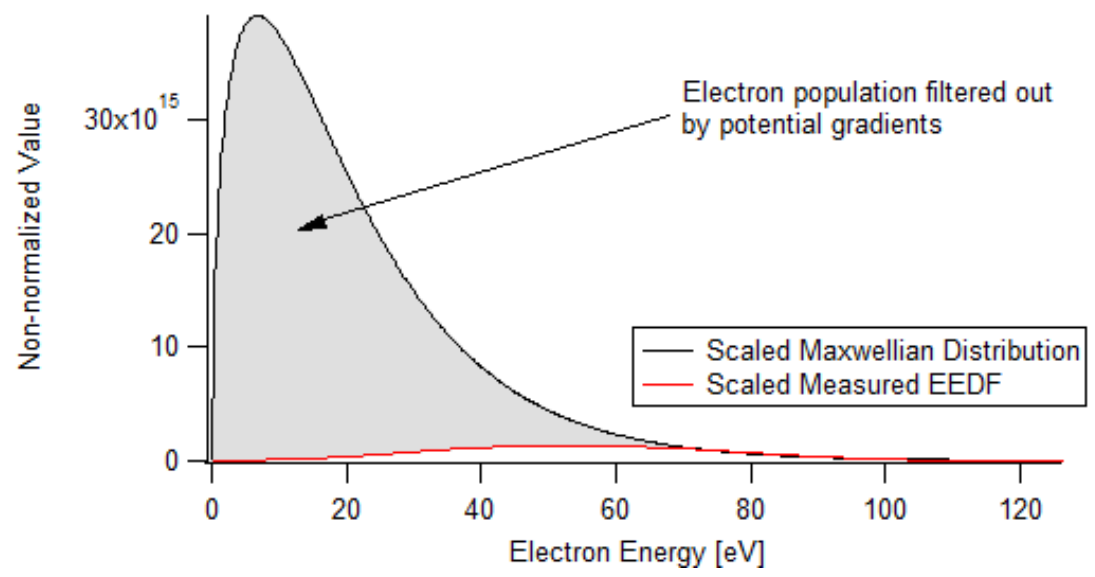

Figure 12: Comparison of a measured EEDF to the predicted Maxwellian distribution based on the measured electron temperature. The measured EEDF is scaled to the local density while the Maxwellian distribution is scaled to a density closer to the channel centerline (along the same magnetic field line).

\section{Conclusion}

A flush-mounted Langmuir probe and swept emissive probe were used to interrogate the near-wall region by the exit plane of a 6-kW Hall thruster under a variety of operating conditions. Sheridan's model [13] was used to successfully recover the true ion saturation current by correcting for sheath expansion, and the derivative method for obtaining plasma potential from Langmuir probe data was found to be satisfactory when compared to data from the swept emissive probe. When compared to internal measurements taken by Reid et al. [11], it was found that the near-wall region was characterized by a significantly lower density and potential than expected, while the electron temperature was found to be fairly consistent.

Calculated ion power deposition was found to be maximized at low discharge voltage and high flow rate, when discharge currents and divergence are the highest. The changes in sheath potential drop did not seem to have a significant effect on the power deposition. The local EEDF was found to be highly non-Maxwellian, shifting towards higher energies than expected based purely on the measured electron temperatures. It is possible that the large potential gradients associated with the near-wall region are filtering out low-energy electrons, and collisions are infrequent enough that the low-energy spectrum is not replenished. Further measurements need to be made in order to fully characterize the near-wall region, but flush probes appear to be a promising diagnostic to determine the properties relevant to erosion and electron mobility mechanisms.

\section{Acknowledgements}

The authors would like to thank Drs. John Foster and Bryan Reid for fruitful discussion and invaluable input, and David Huang for help in running the Hall thruster used in this study. The authors would also like to acknowledge and thank the Air Force Office of Scientific Research (AFOSR) for funding this research. Dr. Mitat Birkan is the Project Manager for Grant FA9550-06-1-0105. The lead author is supported under the Graduate Student Research Program through the National Aeronautics and Space Administration.

Portions of this research were carried out at the Jet Propulsion Laboratory, California Institute of Technology, under a contract with the National Aeronautics and Space Administration. 


\section{References}

[1] Hofer, R. R., Katz, I., Mikellides, I. G., Goebel, D. M., Jameson, K. K. et al., "Efficacy of Electron Mobility Models in Hybrid-PIC Hall Thruster Simulations," Presented at the 44th AIAA/ASME/SAE/ASEE Joint Propulsion Conference and Exhibit, AIAA-2008-4924, Hartford, CT, July 20-23, 2008.

[2] Hofer, R. R., Mikellides, I. G., Katz, I., and Goebel, D. M., "BPT-4000 Hall Thruster Discharge Chamber Erosion Model Comparison with Qualification Life Test Data," Presented at the 30th International Electric Propulsion Conference, IEPC-2007-267, Florence, Italy, September 17-20, 2007.

[3] Yim, J. T., Keidar, M., and Boyd, I. D., "A Hydrodynamic-Based Erosion Model for Hall Thrusters," Presented at the 29th International Electric Propulsion Conference, IEPC-2005-013, Princeton, NJ, October 31 - November 4, 2005.

[4] Ahedo, E. and Parra, F. I., "Partial trapping of secondary-electron emission in a Hall thruster plasma," Physics of Plasmas 12, 7, 073503-073501 - 073503-073507 (2005).

[5] Barral, S., Makowski, K., Peradzynski, Z., Gascon, N., and Dudeck, M., "Wall material effects in stationary plasma thrusters. II. Near-wall and in-wall conductivity," Physics of Plasmas 10, 10, 4137-4152 (2003).

[6] Keidar, M., Boyd, I. D., and Beilis, I. I., "Plasma flow and plasma-wall transition in Hall thruster channel," Physics of Plasmas 8, 12, 5315-5322 (2001).

[7] Azziz, Y., Warner, N. Z., Martinez-Sanchez, M., and Szabo, J. J., "High Voltage Plume Measurements and Internal Probing of the BHT-1000 Hall Thruster," Presented at the 40th AIAA/ASME/SAE/ASEE Joint Propulsion Conference and Exhibit, AIAA-2004-4097, Fort Lauderdale, FL, July 11-14, 2004.

[8] Kim, V., Grdlichko, D., Kozlov, V., Lazourenko, A., Popov, G. et al., "Local Plasma Parameter Measurements by Nearwall Probes Inside the SPT Accelerating Channel Under Thruster Operation with Kr," Presented at the 38th AIAA/ASME/SAE/ASEE Joint Propulsion Conference and Exhibit, AIAA-2002-4108, Indianapolis, IN, July 7-10, 2002.

[9] Kim, V., Kozlov, V., Skrylnikov, A., Umnitsin, L. N., Svotina, V. V. et al., "Investigation of the Local Plasma Parameters in the SPT Accelerating Channel Under Increased Discharge Voltages," Presented at the 29th International Electric Propulsion Conference, IEPC-2005-004, Princeton, NJ, October 31 - November 4, 2005.

[10] Szabo, J. J., Warner, N. Z., and Martinez-Sanchez, M., "Instrumentation and Modeling of a High Isp Hall Thruster," Presented at the 38th AIAA/ASME/SAE/ASEE Joint Propulsion Conference and Exhibit, AIAA-2002-4248, Indianapolis, IN, July 7-10, 2002.

[11] Reid, B. M., "The Influence of Neutral Flow Rate in the Operation of Hall Thrusters," Ph.D. Dissertation, Aerospace Engineering, University of Michigan, 2008.

[12] Hershkowitz, N., in Plasma Diagnostics: Discharge Parameters and Chemistry, edited by Flamm, D. L. (Academic Press, Inc., 1989), pp. 113-181.

[13] Sheridan, T. E., "How big is a small Langmuir probe?," Physics of Plasmas 7, 7, 3084-3088 (2000).

[14] Druyvesteyn, M. J.," Z. Phys. 64, 781 (1930).

[15] Haas, J. M., "Low-Perturbation Interrogation of the Internal and Near-Field Plasma Structure of a Hall Thruster Using a High-Speed Probe Positioning System," Ph.D. Dissertation, Aerospace Engineering, University of Michigan, 2001.

[16] Linnell, J. A., "An Evaluation of Krypton Propellant in Hall Thrusters," Ph.D. Dissertation, Aerospace Engineering, University of Michigan, 2007.

[17] Hershkowitz, N. and Cho, M. H., "Measurement of plasma potential using collecting and emitting probes," The Journal of Vacuum Science and Technology A 6, 3, 2054-2059 (1988). 
[18] Hofer, R. R., "Development and Characterization of High-Efficiency, High-Specific Impulse Xenon Hall Thrusters," Ph.D Dissertation, Aerospace Engineering, The University of Michigan, 2004.

[19] Hofer, R. R., Mikellides, I. G., Katz, I., and Goebel, D. M., "Wall Sheath and Electron Mobility Modeling in HybridPIC Hall Thruster Simulations," Presented at the 43rd AIAA/ASME/SAE/ASEE Joint Propulsion Conference and Exhibit, AIAA-2007-5267, Cincinnati, OH, July 8-11, 2007. 